\title{
Multiple pregnancy after single or multiple embryo transfer performed according to Korean guidelines
}

\author{
E Jung Han', Seul Ki Kim', Jung Ryeol Lee ${ }^{1,2}$, Byung Chul Jee ${ }^{1,2}$, Chang Suk Suh ${ }^{1,2,3}$, Seok Hyun Kim²,3 \\ 'Department of Obstetrics and Gynecology, Seoul National University Bundang Hospital, Seoul National University College of Medicine, Seongnam; \\ 2Department of Obstetrics and Gynecology, Seoul National University College of Medicine, Seoul; ${ }^{3}$ Department of Obstetrics and Gynecology, Seoul \\ National University Hospital, Seoul National University College of Medicine, Seoul, Korea
}

Objective: To assess compliance with Korean guidelines for embryo transfer, the possible reasons for non-compliance, and multiple pregnancies according to each specific condition in compliant cycles.

Methods: A single-institution, retrospective study was conducted of 256 fresh in vitro fertilization cycles during 2012-2014. To assess compliance with Korean guidelines, the maximum recommended number of embryos transferred (according to criteria of age, transfer day, and presence of favorable conditions) was compared with the actual number of embryos transferred. Clinical pregnancy rate (PR) was assessed as the percentage of pregnant women resulting from each set of transfer conditions, including the number of embryos transferred. The multiple pregnancy rate (MPR) was calculated as the percentage of pregnant women with a multifetal pregnancy.

Results: The compliance rate with the Korean guidelines was $96.5 \%$ (247/256). Non-compliance occurred in nine cycles owing to poor embryo quality, repeated implantation failure, or hostile endometrium. In compliant cycles, the PR was $31.2 \%$ (77/247), and the MPR was 27.3\% (21/77; 20 twins and one triplet). Higher MPR was noted in two types of transfer conditions: transfer of three cleavage embryos in women aged 35-39 years with favorable conditions (66.7\%; primarily from those aged 35-37 years) and transfer of two blastocysts in women aged $\geq 40$ years with favorable conditions (50\%).

Conclusion: Under the Korean guidelines, compliance rate was high in our center. Multiple pregnancies occurred primarily in group with favorable conditions. In high-risk groups for multiple pregnancies, reducing number of embryos transferred should be considered than suggested in the guideline.

Keywords: Embryo transfer; Guidelines; Multiple pregnancy

\section{Introduction}

In the past 20 years, multifetal births have increased 1.9-fold in Korea and continue to increase steadily, a change that is attributed to

Received: Aug 17, 2015 · Revised: Sep 8, 2015 · Accepted: Oct 20, 2015 Corresponding author: Byung Chul Jee

Department of Obstetrics and Gynecology, Seoul National University Bundang Hospital, Seoul National University College of Medicine, 82 Gumi-ro 173beongil, Bundang-gu, Seongnam 13620, Korea

Tel:+82-31-787-7254 Fax:+82-31-787-4054 E-mail: blasto@snubh.org

*This work was supported by a grant (No. A120043) from the Korea Health Care Technology R\&D Project, Ministry of Health and Welfare, Korea.

This is an Open Access article distributed under the terms of the Creative Commons Attribution Non-Commercial License (http://creativecommons.org/licenses/by-nc/3.0/) which permits unrestricted non-commercial use, distribution, and reproduction in any medium, provided the original work is properly cited. the increasing use of assisted reproductive technology [1,2]. In medically assisted reproduction, multiple ovulation induced by ovarian stimulation and multiple embryo transfer in in vitro fertilization (IVF) cycles commonly result in multifetal pregnancy. It is well known that multifetal pregnancies can cause obstetric complications such as preterm births and low birth weight, thus increasing maternal and fetal morbidity and mortality [3]. In a recent meta-analysis, multifetal pregnancies generated by assisted reproduction were associated with higher risks of pregnancy-related complications and adverse pregnancy outcomes than those arising from spontaneous conception [4].

The issues regarding multifetal pregnancy in IVF cycles have been emerging since the late 1990's from Europe and Australia. Several countries have law or guidelines to regulate the number of embryos 
Table 1. Recommended limits on the numbers of embryos to transfer

\begin{tabular}{|c|c|c|c|c|c|c|c|c|c|}
\hline \multicolumn{5}{|c|}{ USA } & \multicolumn{5}{|c|}{ Korea $^{\text {b) }}$} \\
\hline \multirow{2}{*}{ Age (yr) } & \multicolumn{2}{|c|}{ Cleavage stage } & \multicolumn{2}{|c|}{ Blastocysts } & \multirow{2}{*}{ Age (yr) } & \multicolumn{2}{|c|}{ Day 2-3 transfer } & \multicolumn{2}{|c|}{ Day 5-6 transfer } \\
\hline & Favorable & All others & Favorable & All others & & Favorable & Not favorable & Favorable & Not favorable \\
\hline$<35$ & $1-2$ & 2 & 1 & 2 & $<35$ & 2 & 3 & $1-2$ & 2 \\
\hline $35-37$ & 2 & 3 & 2 & 2 & $35-39$ & 3 & 4 & 2 & 3 \\
\hline $38-40$ & 3 & 4 & 2 & 3 & & & & & \\
\hline$>40$ & 5 & 5 & 3 & 3 & $\geq 40$ & 5 & 5 & 3 & 3 \\
\hline
\end{tabular}

a) Favorable = first in vitro fertilization (IVF) cycle, good embryo quality, excess embryos available for cryopreservation, or previous successful IVF cycle; b) Favorable = first IVF cycle, good embryo quality, excess embryos available for cryopreservation, or previous successful IVF cycle. Good embryo quality indicates $\geq 4$-cell with $\geq$ grade $B$ at day $2, \geq 6$-cell with $\geq$ grade $B$ at day $3, \geq 8$-cell with $\geq$ grade $B$ at day 4 , any blastocyst at day 5 , or a blastocyst with $\geq 50 \%$ blastocele at day 6 . Transfer where good and poor quality embryos are mixed is considered to be unfavorable conditions.

transferred. In USA, guidelines for the number of embryos transferred were established in 1998 by the American Society for Reproductive Medicine/Society for Assisted Reproductive Technology (ASRM/SART) and revised in 2004 [5]. According to these guidelines, the number of embryos transferred depends on three criteria: maternal age, transfer day, and the presence of favorable conditions [5]. The USA guidelines were further revised in 2006 by the addition of criteria for blastocyst transfer [6], but the number of embryos transferred remained unchanged in the most recently published revision in 2013 (Table 1) [7].

In Korea, guidelines for the number of embryos transferred were established in 2008 by the government. The three criteria and four favorable conditions are similar to those in the USA guidelines (Table 1), but with differences as follows: (1) maternal age is divided into three groups (compared to four in the USA); (2) a maximum recommended number of three cleavage embryos is transferred in women aged $<35$ years with non-favorable conditions (compared to two embryos in the USA), or two blastocysts with favorable conditions (compared to one in the USA); and (3) the maximum recommended numbers of embryos transferred in women aged 35-39 years is the same as that in American women aged $38-40$ years (i.e., one additional embryo can be transferred in Korean women aged 35-37 years than in American women of this age).

Taken together, the recommended maximum number of embryos transferred according to the Korean guidelines is higher than in the USA guidelines. The present study aimed to investigate whether the Korean guidelines are appropriate for reducing the number of multiple pregnancies. The frequency of compliant cycles was assessed and the reasons for non-compliance were explored. In compliant cycles, the pregnancy rate (PR) and multiple pregnancy rate (MPR) were assessed according to each transfer condition.

\section{Methods}

A single-institution, retrospective study was conducted at the Seoul
National University Bundang Hospital with the approval of the Institutional Review Board. Two hundred and fifty-six fresh IVF cycles with embryo transfer performed between 2012 and 2014 were identified. Patient demographics and clinical characteristics were collected through electronic medical records, which included maternal age, parity, cycle number, transfer day, and number of embryos transferred.

Information on embryo quality assessed by cell number and grade was also collected. The grade of embryo was evaluated using morphological criteria, based on the degree of fragmentation and the regularity of blastomeres, as follows: grade A, equal-sized blastomeres and no fragments or apparent morphologic abnormalities; grade $B$, equal-sized blastomeres and $<20 \%$ of fragments without apparent morphologic abnormalities; grade $\mathrm{C}$, irregularity of blastomeres and $20 \%-50 \%$ of fragments without apparent morphologic abnormalities; grade D, irregularity of blastomeres and $>50 \%$ fragments with apparent morphologic abnormalities.

Clinical pregnancy was defined as the detection of identifiable intrauterine gestational sac(s) with fetal heartbeat(s), and PR was calculated as the percentage of pregnant women per transfer cycle. Multifetal pregnancy was defined as the detection of $\geq 2$ intrauterine gestational sacs with fetal heartbeats, and the MPR was calculated as the percentage of pregnant women with multifetal pregnancy. The initial number of gestational sac(s) was always used in analysis, although subsequent miscarriage or vanishing twin sometimes occurred.

The maximum recommended number of embryos transferred was assessed and compared to the actual number of embryos transferred, and compliance or non-compliance with the recommendation was determined. In non-compliant cycles, the reasons for noncompliance and the occurrence of any resultant pregnancy were recorded. In compliant cycles, PR and MPR were assessed according to each specific transfer condition and the number of embryos transferred. The present study was a simple descriptive study; therefore, no statistical analysis was needed. 


\section{Results}

At our institution, 256 fresh IVF cycles were performed from 20122014. The compliance rate was $96.5 \%$ (247/256), with non-compliance in nine cycles. Reasons for non-compliance were poor embryo quality in four cycles, repeated implantation failure in four cycles, and hostile endometrium in one cycle (Table 2). In the non-compliant group, five pregnancies occurred ( $P R=55.6 \%)$ and one twin pregnancy was identified among these (MPR $=20 \%$ ).

In the compliant group (247 cycles), the mean age of the women was 35.9 years and the mean number of embryos transferred was two (one embryo in 60 cycles, two in 150 cycles, three in 31 cycles, and four in 6 cycles). Day 2 transfer was performed in two cycles, day 3 transfer in 196 cycles, day 4 transfer in one cycle, and day 5 transfer in 48 cycles. The overall PR in the compliant group was $31.2 \%$ (77/247), the MPR was $27.3 \%$ (21/77; 20 twins and 1 triplet), and the implantation rate was $20.8 \%$ (99/477). Spontaneous miscarriage was identified in 17 women (miscarriage rate $=22.1 \%$ [17/77]).

One case of triplet pregnancy occurred in a 36-year-old, nulliparous woman. She underwent her second IVF cycle and good quality em- bryos were obtained (one 8-cell embryo of grade A and two 8-cell embryos of grade B); thus, the maximum recommended number of embryos was three at day 3 . Three embryos were transferred at day 3 (compliant cycle), and a triplet pregnancy was subsequently noted. She underwent selective fetal reduction and the resulting twins were delivered via cesarean section.

Table 3 shows the PR and MPR according to the number of embryos transferred, irrespective of age and transfer day. Interestingly, twin pregnancies also occurred after single embryo transfer (MPR $=6.7 \%$ ).

Table 4 shows the PR and MPR for each transfer condition in the compliant group (247 cycles). Higher MPR was noted in two situations with the presence of favorable conditions: transfer of three cleavage embryos in women aged $35-39$ years (66.7\%) and transfer of two blastocysts in women aged $\geq 40$ years (50\%).

When the group aged $35-39$ years was divided into those aged 35 37 years and $38-39$ years, multiple pregnancies from transfer of three cleavage embryos and transfer of two blastocysts were only seen in the group aged 35-37 years (Table 5). Under the same transfer conditions, no pregnancies occurred in the group aged $38-39$ years.

Table 2. Summary of nine non-compliant cycles

\begin{tabular}{|c|c|c|c|c|c|c|c|c|}
\hline Year & $\begin{array}{l}\text { Age } \\
\text { (yr) }\end{array}$ & $\begin{array}{c}\text { Cycle } \\
\text { no. }\end{array}$ & $\begin{array}{l}\text { Favorable } \\
\text { conditions }\end{array}$ & $\begin{array}{l}\text { Max no. embryos } \\
\text { recommended }\end{array}$ & $\begin{array}{l}\text { Actual no. embryos } \\
\text { transferred }\end{array}$ & $\begin{array}{c}\text { Reason for } \\
\text { non-compliance }\end{array}$ & Pregnancy & $\begin{array}{c}\text { Multiple } \\
\text { pregnancy }\end{array}$ \\
\hline 2014 & 34 & 1 & 1st cycle & 2 & 3 & Poor $(12 B, 6 C, 6 C)$ & No & - \\
\hline 2013 & 33 & 5 & Good (8B, 8B, 6B) & 2 & 3 & RIF & $Y_{e s}{ }^{a)}$ & No \\
\hline 2013 & 32 & 1 & 1st cycle, Good (8A, 8B, 8B) & 2 & 3 & Thin endometrium & No & - \\
\hline 2013 & 31 & 1 & 1st cycle & 2 & 3 & Poor $(8 \mathrm{~A}, 5 \mathrm{~A}, 4 \mathrm{~B})$ & Yes & No \\
\hline 2012 & 28 & 4 & Good (8A, 8B, 8B), Cryo & 2 & 3 & RIF & No & - \\
\hline 2012 & 28 & 1 & 1st cycle & 2 & 3 & Poor $(8 B, 5 B, 5 C)$ & $Y_{e s}{ }^{a)}$ & No \\
\hline
\end{tabular}

All cycles were day 3 transfer.

Good, good embryo quality; Poor, poor embryo quality; Cryo, surplus embryos for cryopreservation; RIF, recurrent implantation failure.

a)Ended in miscarriage.

Table 3. Frequency of number of embryos transferred, PR, and MPR from our institution and from Korean registry data

\begin{tabular}{|c|c|c|c|c|c|c|c|c|c|}
\hline \multirow{3}{*}{ No. of embryos } & \multicolumn{6}{|c|}{ From our institution (2012-2014) } & \multirow{2}{*}{\multicolumn{3}{|c|}{ From Korean data (2010) }} \\
\hline & \multicolumn{3}{|c|}{ Compliance group } & \multicolumn{3}{|c|}{ All study groups } & & & \\
\hline & Frequency (\%) & PR (\%) & MPR (\%) & Frequency (\%) & PR (\%) & MPR (\%) & Frequency (\%) & PR (\%) & MPR (\%) \\
\hline 1 & 24.3 & 25.0 & 6.7 & 23.4 & 25.0 & 6.7 & 14.0 & 19.1 & 0.5 \\
\hline 2 & 60.7 & 38.0 & 29.8 & 58.6 & 38.0 & 29.8 & 36.3 & 35.9 & 24.8 \\
\hline 3 & 12.6 & 16.1 & 40.0 & 15.2 & 23.1 & 33.3 & 37.3 & 32.9 & 28.9 \\
\hline 4 & 2.4 & 0 & - & 2.7 & 14.3 & 0 & 10.3 & 31.1 & 33.4 \\
\hline 5 & - & - & - & - & - & - & 2.1 & 23.3 & 36.1 \\
\hline$\geq 6$ & - & - & - & - & - & - & 0.1 & 13.0 & 66.9 \\
\hline
\end{tabular}

See reference [8] for Korean data (2010).

$\mathrm{PR}$, pregnancy rate; MPR, multiple pregnancy rate. 
Table 4. Pregnancy rate and multiple pregnancy rate according to each specific transfer condition

\begin{tabular}{|c|c|c|c|c|c|c|c|}
\hline \multirow{2}{*}{ Age (yr) } & \multicolumn{4}{|c|}{ Day 2-4 transfer } & \multicolumn{3}{|c|}{ Day 5 transfer } \\
\hline & No. & Favorable & No. & Not favorable & No. & Favorable & Not favorable \\
\hline \multicolumn{8}{|l|}{$<35$} \\
\hline 1 & 15 & $13.3 / 0$ & 4 & $25.0 / 0$ & 6 & $66.7 / 25.0$ & No case \\
\hline 2 & 41 & $34.1 / 35.7$ & 6 & $66.7 / 25.0$ & 23 & $43.5 / 40.0$ & No case \\
\hline 3 & - & - & 7 & $14.3 / 0$ & - & - & - \\
\hline \multicolumn{8}{|l|}{$35-39$} \\
\hline 1 & 12 & $41.7 / 20.0$ & 3 & No pregnancy & 5 & $40.0 / 0$ & No case \\
\hline 2 & 43 & $37.2 / 18.8$ & 6 & $16.7 / 0$ & 10 & $60.0 / 33.3$ & No case \\
\hline 3 & 7 & $42.9 / 66.7$ & 9 & $11.1 / 0$ & - & - & No case \\
\hline 4 & - & - & - & No case & - & - & - \\
\hline \multicolumn{8}{|l|}{$\geq 40$} \\
\hline 1 & 14 & $7.1 / 0$ & 1 & No pregnancy & & No case & No case \\
\hline 2 & 15 & $26.7 / 25.0$ & 3 & No pregnancy & 3 & $66.7 / 50.0$ & No case \\
\hline 3 & 6 & No pregnancy & 1 & No pregnancy & 1 & No pregnancy & No case \\
\hline 4 & 6 & No pregnancy & - & No case & - & - & - \\
\hline 5 & - & No case & - & No case & - & - & - \\
\hline
\end{tabular}

The first digit in the columns indicates number of embryos transferred; ' $n$ ' indicates the number of in vitro fertilization cycles in each transfer condition. The first percentage indicates the pregnancy rate, and the second percentage indicates the multiple pregnancy rate among the clinical pregnancies.

Table 5. Pregnancy rate and multiple pregnancy rate according to each specific transfer condition in women aged 35-37 and 38-39 years

\begin{tabular}{|c|c|c|c|c|c|c|c|}
\hline \multirow{2}{*}{ Age (yr) } & \multicolumn{4}{|c|}{ Day 2-4 transfer } & \multicolumn{3}{|c|}{ Day 5 transfer } \\
\hline & No. & Favorable (\%) & No. & Not favorable (\%) & No. & Favorable (\%) & Not favorable \\
\hline \multicolumn{8}{|l|}{$35-37$} \\
\hline 1 & 7 & $57.1 / 0$ & 3 & No pregnancy & 3 & $66.7 / 0$ & No case \\
\hline 2 & 25 & $44.0 / 27.3$ & 4 & $25.0 / 0$ & 9 & $66.7 / 33.3$ & No case \\
\hline 3 & 4 & $75.0 / 66.7$ & 4 & $25.0 / 0$ & - & - & No case \\
\hline 4 & - & - & - & No case & - & - & - \\
\hline \multicolumn{8}{|l|}{$38-39$} \\
\hline 1 & 5 & $20.0 / 100$ & - & No case & 2 & No pregnancy & No case \\
\hline 2 & 18 & $27.8 / 0$ & 2 & No pregnancy & 1 & No pregnancy & No case \\
\hline 3 & 3 & No pregnancy & 5 & No pregnancy & - & - & No case \\
\hline 4 & - & - & - & No case & - & - & - \\
\hline
\end{tabular}

The first digit in the columns indicates number of embryos transferred; ' $n$ ' indicates the number of in vitro fertilization cycles in each transfer condition. The first percentage indicates the pregnancy rate, and the second percentage indicates the multiple pregnancy rate among the clinical pregnancies.

\section{Discussion}

Regulation of the number of embryos for transfer primarily aims to reduce high-order multifetal births. In our study, only one triplet pregnancy was noted in all cycles, including the compliant and noncompliant groups; clinical pregnancies occurred in 82 cycles $(\mathrm{PR}=32.0 \%)$, including 21 twin and 1 triplet pregnancy (MPR = 26.8\%). Although non-compliance with the transfer guidelines occurred in nine cycles, the PR and the frequency of high-order multiple pregnancy were acceptable. Moreover, selective fetal reduction was performed in the one triplet case, thus multi-fetal births larger than twin births did not occur.

Higher MPR was noted in two situations in the present study; trans- fer of three cleavage embryos in women aged $35-39$ years (66.7\%) and transfer of two blastocysts in women aged $\geq 40$ years. Therefore, it would be reasonable to reduce the number of embryos for transfer than suggested by the guideline in such situations.

Women aged $<35$ years old are a high-risk group for high-order multiple pregnancy. In the Korean guidelines, one additional embryo is permitted to be transferred in women aged $<35$ years, both in day 2-4 transfer of with non-favorable conditions and in day 5-6 transfer with favorable conditions, compared to the USA guidelines. Under these conditions in our population, the MPR was acceptable; thus, reducing the number of embryos for transfer appears to be unnecessary. However, transfer of two blastocysts in women aged $<35$ years with favorable conditions (compliant group) yielded a rather high 
MPR (40\%); therefore, single blastocyst transfer should be considered in that situation.

Maternal age is divided into four groups in the USA guidelines, but in the Korean guidelines, there are only three age groups. The maximum recommended numbers of embryos for transfer in Korean women aged 35-39 years is the same as that in American women aged 38-40 years; thus, one additional embryo can be transferred in Korean women aged 35-37 years. Therefore, according to the Korean guidelines, a higher MPR would be expected in Korean women aged 35-37 years; this was noted to be the case in the present study. The MPR was $66.7 \%$ following transfer of three cleavage embryos in women aged 35-39 years with favorable conditions and multiple pregnancies exclusively emerged from women aged 35-37 years. Therefore, clinicians should consider reducing number of embryos transferred than suggested in the guideline, especially when woman is 35-37 years.

In the USA, an average of two embryos was transferred per cycle in 2010, and the average number of embryos transferred increased with age ( 2.0 among women aged $<35$ years, 2.4 among women aged 35-40 years, and 3.0 among women aged $>40$ years) [9]. In this report, there was a substantial proportion (43.4\%) of twin pregnancies, and a smaller proportion (3.0\%) of triplets or higher order pregnancies. After the publication of the ASRM/SART embryo transfer guidelines, a significant decrease in the number of embryos transferred and multifetal births was reported in the USA $[10,11]$.

In Europe, despite huge differences in embryo transfer policy between countries, the overall trend seen over the last 10 years towards transferring fewer embryos appears to be continuing [12]. In Europe, the most frequent ET number was two between 2008 and 2010, and the percentages of cycles where $\leq 2$ embryos were transferred were $75.6 \%$ in $2008,81.9 \%$ in 2009 , and $82.4 \%$ in 2010 [12]. Among live births, multifetal birth rates were $21.7 \%$ in $2008,20.2 \%$ in 2009 , and $20.6 \%$ in 2010.

In Korea, the embryo transfer number between 2007 and 2008 was mostly three or four $[13,14]$, but this decreased to two or three between 2009 and 2010 [1,8]. Multifetal birth rates were 50.1\% in 2007, $52.2 \%$ in 2008 , and $46.5 \%$ in 2009 , falling to $25.6 \%$ in 2010 $[1,8,13,14]$. The triplet or high-order birth rate was $0.7 \%$ in $2007,1.5 \%$ in $2008,1.2 \%$ in 2009 , and $0.6 \%$ in 2010 . This dramatic decrease in multifetal births in Korea might be due to good compliance with the guidelines for embryo transfer. However, the most frequent transfer number was two or three (overall mean $=2.5$ ) and the percentage of cycles in which $\leq 2$ embryos were transferred was $50.3 \%$ in 2010, a figure that was lower than in Europe.

In the 2013 USA guidelines, in each of the above age groups for patients with $\geq 2$ previous failed fresh IVF cycles or a less favorable prognosis, one additional embryo may be transferred according to individual circumstances [7]. Such documentation is absent in the Korean guidelines, but a statement about clear documentation is included: "Justification for transferring one additional embryo more than the recommended limit should be clearly documented in the patient's medical record." In our study, non-compliance mostly occurred in women with poor embryo quality or repeated implantation failure. Therefore, it is reasonable that one additional embryo is allowed in several exceptional situations, especially in women with a poor prognosis.

In the USA, programs that have a high-order multiple pregnancy ( $\geq 3$ implanted embryos) rate that is $>2$ standard deviations above the mean rate for all SART-reporting clinics, for two consecutive years, may be audited by SART [7]. In Korea, compliance with the guidelines or managing the incidence of high-order multiple pregnancy is not presently regulated.

Reducing the embryo number for transfer is an obvious strategy to reduce high-order multifetal births. As shown in Korean data from 2010, a maximum PR and minimal MPR can be obtained with the transfer of two, rather than three embryos. Therefore, the majority of cases should have two embryos transferred. Reducing the number of embryos transferred in groups at high risk of high-order multiple pregnancy and promotion of elective single embryo transfer is needed to reduce multiple births and the related adverse events of assisted reproductive technology. We recognize that the present study comes from a single institution; thus, further nationwide surveillance is needed to identify groups at high risk of high-order multiple pregnancy.

\section{Conflict of interest}

No potential conflict of interest relevant to this article was reported.

\section{References}

1. Committee for Assisted Reproductive Technology, Korean Society of Obstetrics and Gynecology; Choi YM, Chun SS, Han HD, Hwang $J \mathrm{H}$, Hwang KJ, et al. Current status of assisted reproductive technology in Korea, 2009. Obstet Gynecol Sci 2013;56:353-61.

2. Ha JO. Thirty years of IVF in Korea: the role and responsibility of experts. J Korean Hist Sci Soc 2014;36:63-102.

3. Min JK, Breheny SA, MacLachlan V, Healy DL. What is the most relevant standard of success in assisted reproduction? The singleton, term gestation, live birth rate per cycle initiated: the BESST endpoint for assisted reproduction. Hum Reprod 2004;19: 3-7.

4. Qin J, Wang H, Sheng X, Liang D, Tan H, Xia J. Pregnancy-related complications and adverse pregnancy outcomes in multiple pregnancies resulting from assisted reproductive technology: a 
meta-analysis of cohort studies. Fertil Steril 2015;103:1492-508. e1-7.

5. Practice Committee of the Society for Assisted Reproductive Technology and the American Society for Reproductive Medicine. Guidelines on the number of embryos transferred. Fertil Steril 2004;82 Suppl 1:S1-2.

6. Practice Committee of the Society for Assisted Reproductive Technology and the American Society for Reproductive Medicine. Guidelines on number of embryos transferred. Fertil Steril 2006;86:S51-2.

7. Practice Committee of American Society for Reproductive Medicine; Practice Committee of Society for Assisted Reproductive Technology. Criteria for number of embryos to transfer: a committee opinion. Fertil Steril 2013;99:44-6.

8. Lee GH, Song HJ, Lee KS, Choi YM. Current status of assisted reproductive technology in Korea, 2010. Clin Exp Reprod Med 2015;42:8-13.

9. Sunderam S, Kissin DM, Crawford S, Anderson JE, Folger SG, Jamieson DJ, et al. Assisted reproductive technology surveillance: United States, 2010. MMWR Surveill Summ 2013;62:1-24.

10. Stern JE, Cedars MI, Jain T, Klein NA, Beaird CM, Grainger DA, et al. Assisted reproductive technology practice patterns and the impact of embryo transfer guidelines in the United States. Fertil Steril 2007;88:275-82.

11. Dickey RP. The relative contribution of assisted reproductive technologies and ovulation induction to multiple births in the United States 5 years after the Society for Assisted Reproductive Technology/American Society for Reproductive Medicine recommendation to limit the number of embryos transferred. Fertil Steril 2007;88:1554-61.

12. Kupka MS, Ferraretti AP, de Mouzon J, Erb K, D'Hooghe T, Castilla $J A$, et al. Assisted reproductive technology in Europe, 2010: results generated from European registers by ESHREdagger. Hum Reprod 2014;29:2099-113.

13. Korean Society of Obstetrics and Gynecology. Current status of assisted reproductive technology in Korea, 2007. Korean J Obstet Gynecol 2010;53:1052-77.

14. Assisted Reproductive Technology Committee, Korean Society of Obstetrics and Gynecology. Current status of assisted reproductive technology in Korea, 2008. Korean J Obstet Gynecol 2011; 54:741-63. 